\title{
An Active High-Voltage Divider and Phase Shifter
}

\author{
OSKARS PETERSONS, FELlow, IEEe, AND S. P. MEHTA, MEMBER, IEEE
}

\begin{abstract}
An instrument combining the functions of an active high voltage divider and a phase shifter designed to yield small phase-angle uncertainties within $20 \mu \mathrm{rad}$ is described. It is based on a circuit employing a feedback amplifier and a controlled source. The presence of the controlled source greatly reduces the potential for dynamic instabilities of the feedback loop and effectively eliminates the errors associated with the finite gain of the amplifier. Design and construction details and the results of the accuracy evaluation are presented.
\end{abstract}

\section{INTRODUCTION}

$I^{\prime}$ $\mathrm{N}$ tests to determine power losses of large inductive devices such as power transformers and high-voltage inductors (shunt reactors), measurement instruments (power transducers, voltage and current transformers) are required having very low phase-angle uncertainty. Depending upon the power factor of the load that is measured, the phase-angle uncertainty of the measurement system must be maintained in the 10-100- $\mu$ rad range [1], [2].

A commonly used configuration of equipment for measuring power losses consists of three principal parts: 1) an electronic power transducer; 2) an active voltage divider; and 3) a current transformer compensated either electronically or through two-stage construction. To obtain a number of power, voltage, and current ranges, each principal part is usually combined with precision amplifiers. The general complexity of the resulting test system mandates means for verifying its accuracy. Highly accurate standard wattmeters, voltage dividers, and current transformers have been previously developed and described [3]-[7]. In measurements at low power factors where the real power must be measured with an uncertainty of about one percent (but the uncertainty in the real power must be as low as several tens of parts per million when expressed in terms of the apparent power), only the phase-angle uncertainty is critical in the measurement system, thus reducing the requirements on the measuring instruments and calibrating standards.

This paper describes an instrument that can be used as an accurate high-voltage divider or a phase shifter. It was specifically designed to calibrate test systems that measure load losses of power transformers. Since the instrument is intended for applications involving low power factors, the accuracy emphasis is on the phase angle. The instrument is based on a compensated operational ampli-

Manuscript received June 23, 1986. Revised September 10, 1986.

O. Petersons is with the Electrosystems Division, National Bureau of Standards, Gaithersburg, MD 20899.

S. P. Mehta is with ASEA Electric, Inc., Waukesha, WI 53186.

IEEE Log Number 8613436. fier. The compensation scheme involves a controlled source and is particularly attractive for high-voltage divider applications, since compatible high-voltage capacitors are available commercially. A simple modification converts the divider into a $90^{\circ}$ phase shifter.

The originally estimated systematic uncertainty based on the calibration data of the critical passive components was $\pm 14 \mu \mathrm{rad}$ for the voltage divider mode and $\pm 20 \mu \mathrm{rad}$ for the phase shifter mode of operation. A part of this paper describes the verification of this estimate by calibrating the device on system basis.

\section{Operating Principles}

The voltage divider configuration is illustrated in Fig. 1. The usual operational amplifier circuit is modified by the addition of a controlled source $V_{2}$ that is phase-locked with the input source $V_{1}$ and is adjustable in magnitude and phase angle to produce negligible voltage $V_{4}$ at the junction between the two capacitors. A feedback amplifier with relatively low gain compensates for any drift or imperfect adjustment in the controlled source. A null detector $D$ is utilized to monitor $V_{4}$ and, hence, the adjustment of the controlled source.

The ratio of voltages on the two capacitors is the negative reciprocal of the capacitance ratio followed by a correction term as shown [1]:

$$
\begin{aligned}
V_{1} /\left(V_{2}+V_{3}\right)= & -\left(C_{2} / C_{1}\right)\left[1-V_{4} /\left(V_{2}+V_{3}\right)\right. \\
& \left.-\left(C_{1} / C_{2}\right) V_{4} /\left(V_{2}+V_{3}\right)\right]
\end{aligned}
$$

The voltages $V_{3}$ and $V_{4}$ are related through the gain of the amplifier which, in this case, is -100 . The controlled source can be adjusted so that $V_{3} / V_{2}<10^{-4}$. Under such circumstances, the correction term is of the order of 1 ppm or $1 \mu \mathrm{rad}$ and, thus, negligible. The electronic circuit removes any errors due to the finite gain of the amplifier. Also, since the gain of the amplifier is small, dynamic instabilities in the feedback loop present no problems.

The capacitor $C_{1}$ is a gas-insulated, completely shielded precision high-voltage capacitor having a phase defect of $5 \mu \mathrm{rad}$ of smaller. $C_{2}$ is a solid-dielectric capacitor having a capacitance value that is two to four orders of magnitude larger than $C_{1}$. Such capacitors have small but significant phase defects, typically in the 50-100 $\mu$ rad range. Since the three-terminal or transfer capacitance is of interest, the small loss conductance of $C_{2}$ can be compensated as shown in Fig. 2 with a parallel $T$-network having an effective negative conductance. $R$ is adjusted while the entire network containing $C_{2}$ is compared in an impedance 


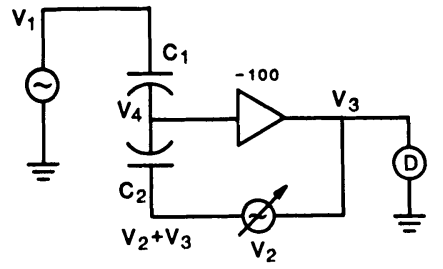

Fig. 1. Active divider with controlled source. $\left(V_{1}\right.$ is the input voltage; $V_{2}$ $+V_{3}$ the output voltage of the divider; $V_{2}$ the voltage of the controlled source; all others are node voltages to ground.)

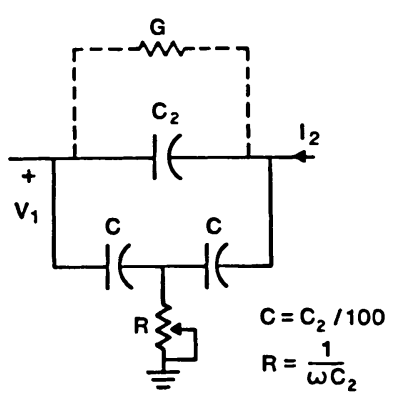

Fig. 2. Low-voltage capacitor network. (The $T$-network compensates the loss of $C_{2}$ represented by the conductance $G$.)

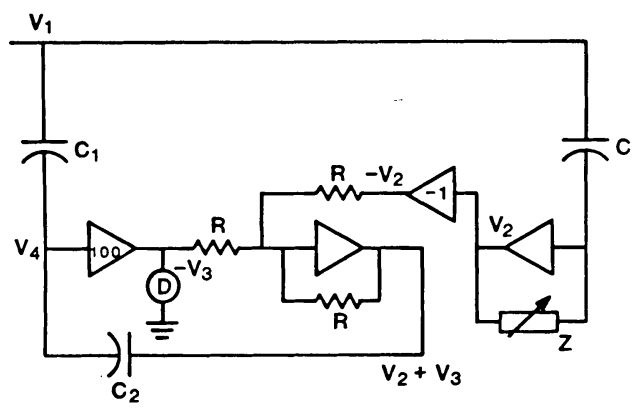

Fig. 3. Schematic diagram of active divider showing the implementation of controlled source ( $V_{2}$ of Fig. 1).

bridge to a gas-dielectric capacitor. This arrangement provides two capacitive networks, $C_{1}$ and $C_{2}$ in Fig. 1, with negligible phase-angle defect. Availability of two such capacitive networks then becomes the only critical requirements in fabricating a divider with negligible phase defect.

There are usually several possibilities for obtaining a phase-locked source for the realization of $V_{2}$ : a tap in the supply transformer, an auxiliary voltage divider, or a separate guard capacitance in the high-voltage capacitor. For this particular instrument the high-voltage capacitor unit has two shielded low-voltage electrodes and, thus, another stable high-voltage capacitor $C$ besides $C_{1}$. The controlled source is instrumented utilizing this extra capacitor and operational amplifier circuitry as shown schematically in Fig. 3. The adjustment of the controlled source has been done manually, but automatic adjustment is possible, for example, by employing a phase-sensitive detector and gain adjustment by means of a digital controller and multiplying digital-to-analog converters.

A $90^{\circ}$ phase shifter for phase-angle calibration of power transducers is implemented in an analogous manner as the

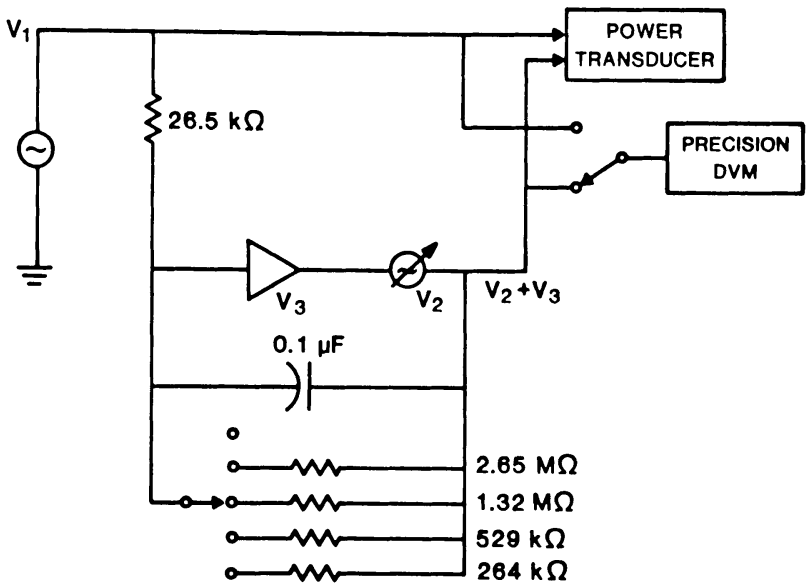

Fig. 4. Schematic diagram of phase shifter and calibration of power transducer. (By deleting the feedback resistors or by connecting one of them in the circuit, phase angles corresponding to power factors of $0,0.01$, $0.02,0.05$, and 0.1 are generated.)

voltage divider: instead of $C_{1}$ and $C$ in Fig. 3, two resistors are used, one of which must have negligible phase defect. The latter requirement is readily achieved with stable miniature resistors installed in a shielded enclosure. The lossless feedback capacitor can be degraded with resistors in parallel as shown in Fig. 4 to yield certain specific power factors: $0.01,0.02,0.05$, and 0.1 , in addition to zero. It should be emphasized that only the input resistor and the feedback capacitor must be precision components with respect to their phase defects. The phase shifter, unlike the voltage divider, is low-voltage instrument. Calibration of a "power transducer" (actually a voltage multiplier) is illustrated in Fig. 4. The phase shifter of Fig. 4 provides negative phantom power to the transducer. Connecting a unity-gain inverting amplifier in cascade with the phase shifter provides positive power. In addition, the combined instrument is useful in self-calibration mode as described later.

\section{Design and Construction Details}

Fig. 5 is the circuit diagram of the active divider/phase shifter including the external precision components $C_{1}$, $C_{2}$, and $C$ that correspond to those in Fig. 3. The amplifier $A 1$ is the main amplifier with a gain of $100 ; A 3, A 4$, and $A 5$ constitute the controlled source; $A 2$ is the summing amplifier adding the outputs of the main amplifier and the controlled source and $A 6$ and $A 7$, together with the capacitors at their inputs, serve for blocking dc offsets that are amplified by $A 1$ and $A 5$. Some compensation in the frequency response and amplitude limiting had to be introduced at the input to $A 7$ to ensure dynamic stability. The voltages $V_{1}, V_{2}, V_{3}$, and $V_{4}$ correspond to those in Fig. 3.

The amplified summing point voltage can be monitored with a null detector or an oscilloscope either directly at BNC3 or after tuned amplification at BNC4. The summing point voltage is adjusted by means of the variable resistors in the feedback loop of $A 4$. 


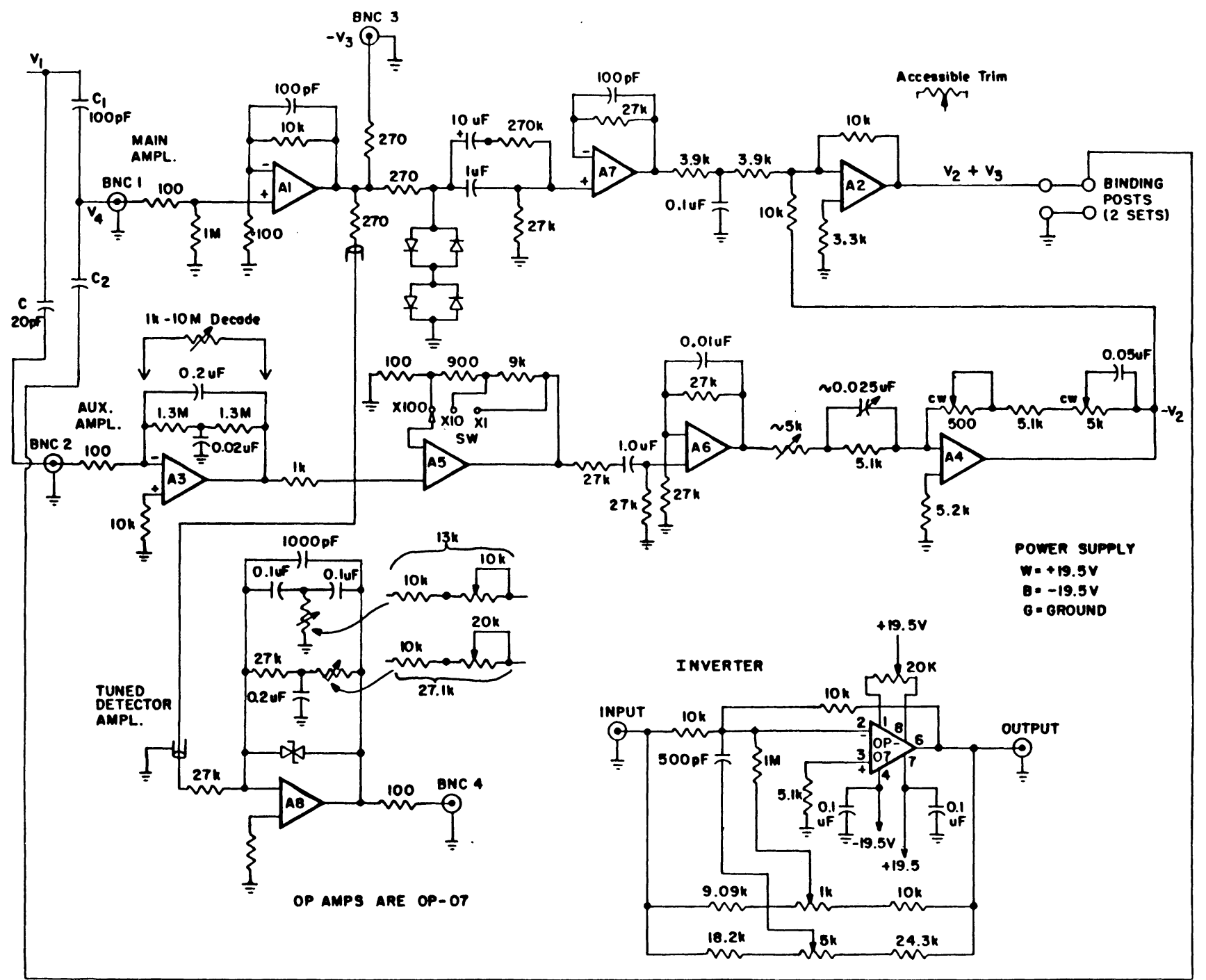

Fig. 5. Detailed circuit diagram of the active divider. (External precision components $C_{1}, C_{2}$, and $C$, corresponding to those in Fig. 3, are also shown.)

The principal passive precision componets are: a dual $100-\mathrm{pF} / 20-\mathrm{pF}$ gas-dielectric high-voltage capacitor for the input; and three solid-dielectric capacitors, $0.01 \mu \mathrm{F}$, $0.1 \mu \mathrm{F}$, and $1.0 \mu \mathrm{F}$, for the feedback loop. Since the feedback capacitors are not changed in the auxiliary amplifier, its gain is made adjustable over three decades. Of course, another pair of capacitors having the capacitance ratio of $5: 1$ can be used for input.

In the phase-shifter application, two resistors having the values of $26.5 \mathrm{k} \Omega$ and $132.5 \mathrm{k} \Omega$, respectively, are used for the input. The appropriate power factors are obtained by using resistors in the feedback loop as indicated in Fig. 4. The phase angle in the auxiliary amplifier is shifted by means of a decade resistor in the feedback of $A 3$.

The inverter that must be cascaded to the amplifier to generate positive losses is shown in the lower right portion of Fig. 5. Provisions are made to trim the inverter to eliminate both the in-phase and quadrature corrections. The procedure is identical to that described later for the entire active divider and is illustrated in Fig. 6(a).

\section{ACCURACY Verification}

The design philosophy of the active divider and the phase shifter was to ensure their accuracy through the calibration of passive components, and to monitor the performance of the amplifier at its summing point. A systembased verification is still useful in order to detect possible sources of error which might not be obvious from the tests on individual components. Pickup of extraneous signals by the sensitive parts of the active divider, layout of connecting leads, and insidious defects in grounding could produce errors that may not be observed in the passive components.

The calibration and adjustment of the standard capacitors has been discussed previously [1]. In approximately two years since the original calibration, no changes have been observed in the phase angles of capacitors. The limits of phase-angle defect of the critical resistors is estimated from the geometrical considerations of the resistors and their enclosures. 
TABLE I

TESTS OF ACTIVE Divider

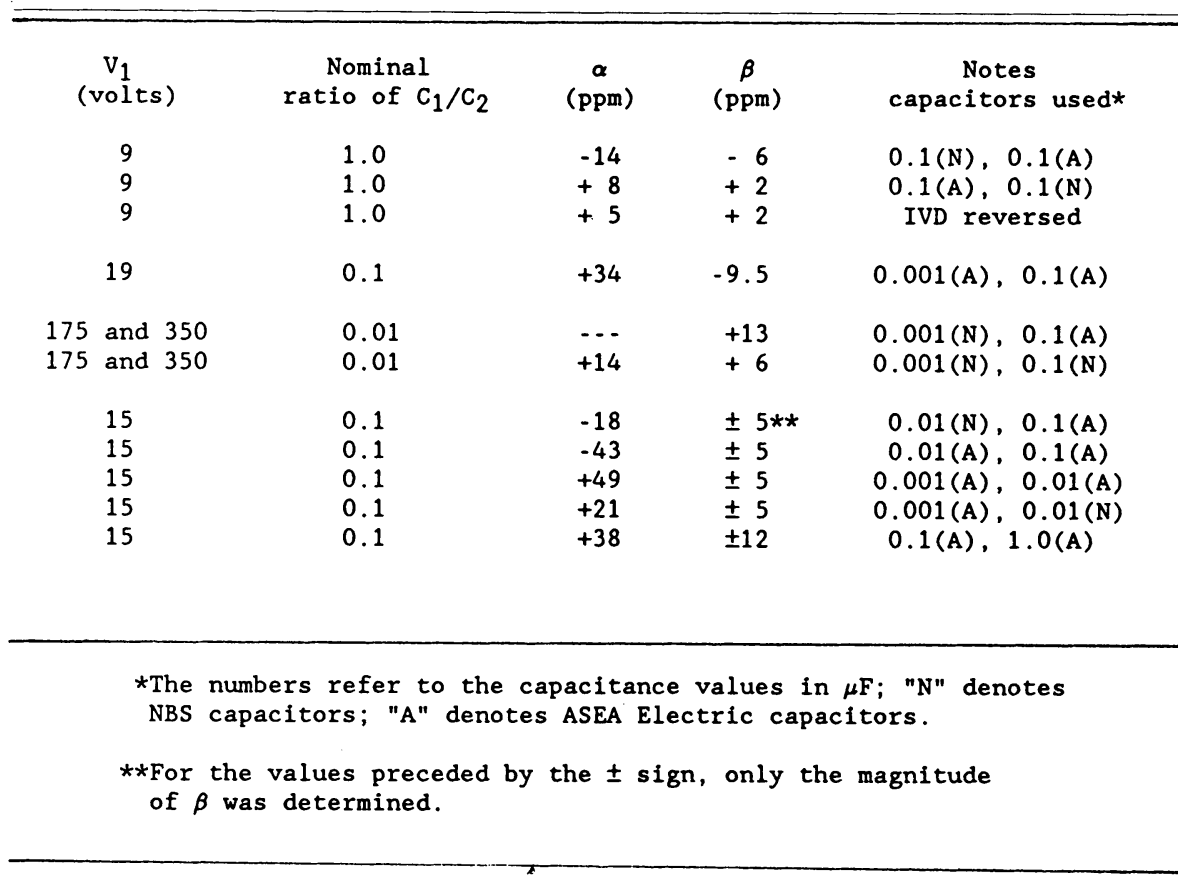

\section{A. Active Divider}

Two circuits were utilized to check the divider at the ratios of $1: 1,1: 10$, and $1: 100$. These circuits, Fig. 6 , employ as reference dividers a multidecade inductive divider in one case and a passive capacitive divider in the other case. The resultant bridge circuits are balanced for the in-phase component only. During a part of the tests a phase-sensitive detector was used which enabled us to obtain the sign for the quadrature or the phase-angle correction. For some of the tests, only the magnitude of the quadrature correction was measured by using a null detector.

The test results are given in Table I. The voltage ratio of the divider is expressed, using the symbols in Fig. 6, as

$$
V_{2} / V_{1}=-\left(C_{1} / C_{2}\right)(1+\alpha+j \beta)
$$

where $C_{1} / C_{2}$ is the actual capacitance ratio obtained from the calibration of the capacitors, and $\alpha$ and $\beta$ are the inphase and quadrature corrections, respectively. In the cases where only the magnitude of $\beta$ was determined, the value in Table $I$ is preceded by the \pm sign.

As noted from the results, in most cases the quadrature error is within $10 \mathrm{ppm}$ as expected from the calibration of the components involved in the circuits of Fig. 6 . In the three cases where the correction is near or above $10 \mathrm{ppm}$, the likely causes of error were identified as extraneous signals. The in-phase corrections are larger, but within $100 \mathrm{ppm}$. This value is consistent with the expected stabilities of solid-dielectric capacitors. All corrections are within the requirements of the application for which the divider was designed.

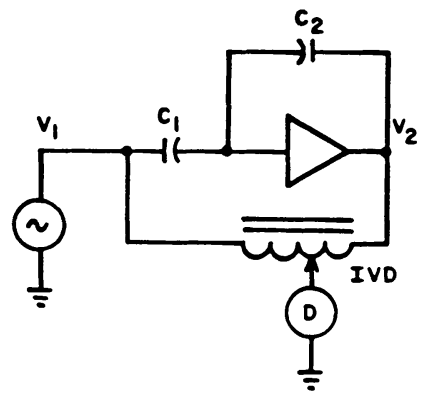

(a)

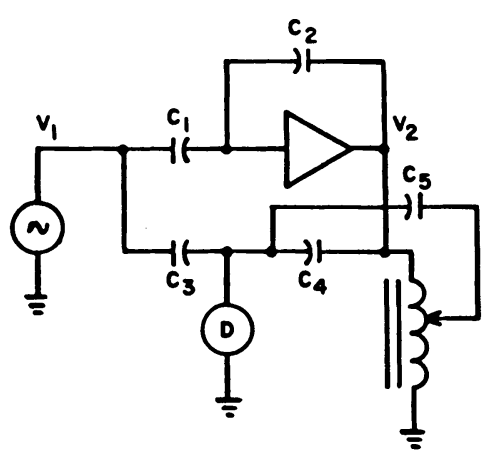

(b)

Fig. 6. Calibration of active voltage divider (a) against inductive divider; (b) against passive capacitive divider.

\section{B. Phase Shifter and Inverter}

The phase shifter, both with and without the inverter, was tested using the two-circuits in Fig. 7: against a passive $R C$ network and against a phase-angle standard that is based on digital synthesis technique [7]. The input and output voltages in the circuit of Fig. 7(a) are related by

$$
V_{2} / V_{1}=j\left(1 / \omega R_{1} C_{2}\right)(1+\alpha+j \beta) \text {. }
$$


TABLE II

Tests OF PHASE ShifTER/INVERTER AT $\pm 90^{\circ}$

\begin{tabular}{|c|c|c|c|}
\hline $\begin{array}{c}\mathrm{v}_{1} \\
\text { (volts) }\end{array}$ & $\begin{array}{c}\text { Nominal } \\
\text { phase angle }\end{array}$ & $\begin{array}{c}\beta \\
(\mathrm{ppm})\end{array}$ & $\begin{array}{l}\text { Notes } \\
\text { components used } *\end{array}$ \\
\hline 9 & $+90^{\circ}$ & $\pm 3 * *$ & $26.5(\mathrm{~A}), 0.1(\mathrm{~A})$ \\
\hline $\begin{array}{l}9 \\
9\end{array}$ & $\begin{array}{l}+90^{\circ} \\
+90^{\circ}\end{array}$ & $\begin{array}{l}+2 \\
-5\end{array}$ & $\begin{array}{ll}26.5(\mathrm{~A}), & 0.1(\mathrm{~A}) \\
26.5(\mathrm{~A}), & 0.1(\mathrm{~N})\end{array}$ \\
\hline $\begin{array}{l}5 \\
5\end{array}$ & $\begin{array}{l}+90^{\circ} \\
-90^{\circ}\end{array}$ & $\begin{array}{r}0 \\
+3\end{array}$ & $\begin{array}{l}\text { phase standard } \\
\text { phase standard }\end{array}$ \\
\hline \multicolumn{4}{|c|}{$\begin{array}{l}\star 26.5 \text { is the resistance value in kilohms; } 0.1 \text { is the } \\
\text { capacitance value in } \mu \mathrm{F} \text {. "A" denotes ASEA Electric's } \\
\text { components; " } N \text { " denotes NBS' components. } \\
\star * \text { For the value preceded by the } \pm \text { sign, only the magnitude } \\
\text { of } \beta \text { was determined. }\end{array}$} \\
\hline
\end{tabular}

TABLE III

Tests of Phase Shifter/Inverter at Power Factors of 0.01 to 0.1

\begin{tabular}{ccccc}
\hline $\begin{array}{c}\mathrm{V}_{1} \\
\text { (volts) }\end{array}$ & $\begin{array}{c}\mathrm{R}_{2} \\
\text { (megohms) }\end{array}$ & $\begin{array}{c}\text { Generated } \\
\text { power factor }(G)\end{array}$ & $\begin{array}{c}\text { Measured } \\
\text { power factor }(M)\end{array}$ & $\begin{array}{c}\text { Power factor difference } \\
(\mathrm{M}-G)\end{array}$ \\
5 & 2.6338 & $1.0061 \times 10^{-2}$ & $1.0067 \times 10^{-2}$ & $6 \times 10^{-6}$ \\
5 & 1.3190 & $2.0090 \times 10^{-2}$ & $2.0091 \times 10^{-2}$ & $1 \times 10^{-6}$ \\
5 & 0.52782 & $5.0141 \times 10^{-2}$ & $5.0157 \times 10^{-2}$ & $16 \times 10^{-6}$ \\
5 & 0.26308 & $1.0022 \times 10^{-1}$ & $1.0023 \times 10^{-1}$ & $10 \times 10^{-6}$ \\
& & & $C_{2}-1.00103 \mu \mathrm{F}$ & \\
& & &
\end{tabular}

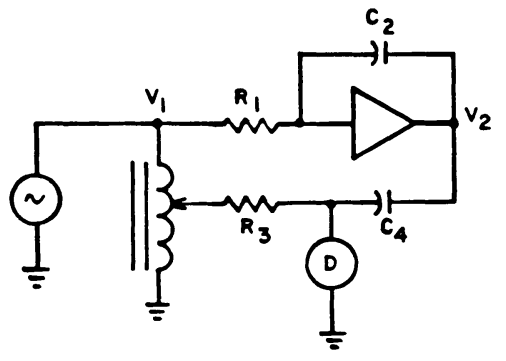

(a)

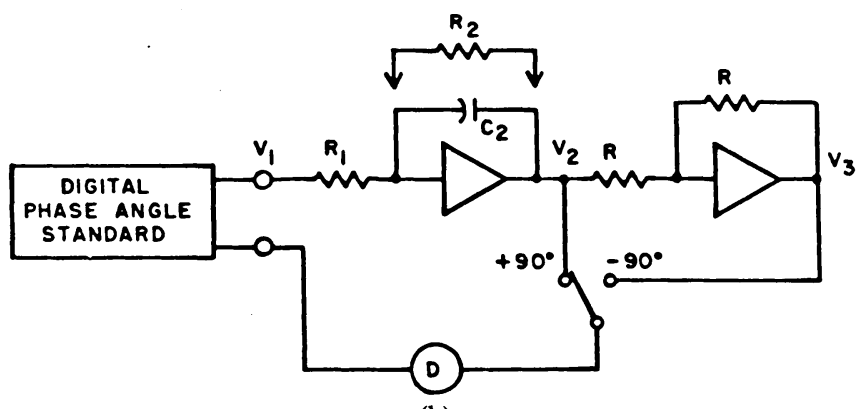

(b)

Fig. 7. Calibration of phase shifter (a) against passive $R C$ network (only at $+90^{\circ}$ ); (b) against digital phase-angle standard (at $\pm 90^{\circ}$ and other phase angles generated by connecting $R_{2}$ in parallel with $C_{2}$ ).
Only the quadrature correction is of interest here. When the instrument is serving as a phase shifter, as illustrated in Fig. 4, the magnitudes of the input and output voltages are measured independently.

For the test points of $\pm 90^{\circ}$, the results are presented in Table II. In the first measurement reported in Table II, only the magnitude of $\beta$ was determined, hence the \pm sign. For the phase angles corresponding to specific power factors of $0.01,0.02,0.05$, and 0.1 , the test data are given in Table III.

The quadrature corrections for all tests at $\pm 90^{\circ}$ are well within $10 \mathrm{ppm}$. At specific power factors the predicted and measured power factor values agree within \pm 0.1 percent of the nominal power factor. In fact the largest phase angle correction is $16 \mu \mathrm{rad}$ in the total phase shift of $50000 \mu \mathrm{rad}$.

\section{Self-Calibration of Phase Shifter and Power Transducer}

Phase-angle measurements are ratio determinations. Hence, self-contained methods, especially at cardinal points of $180^{\circ}$ and $\pm 90^{\circ}$ can be implemented without recourse to higher echelon standards. Using the circuit of 


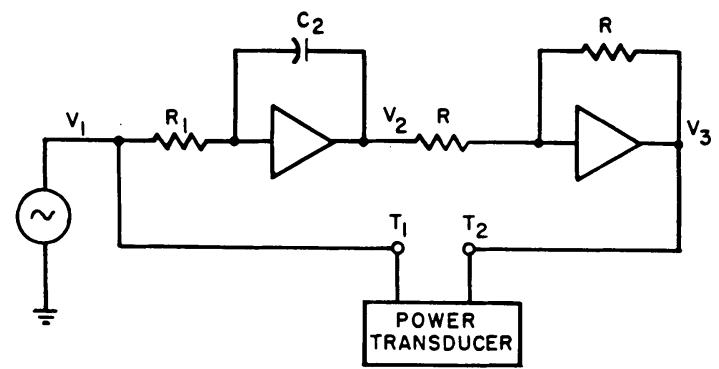

Fig. 8. Self-calibration of phase shifter and power transducer at $\pm 90^{\circ}$ points. (The input terminals of the transducer, $T_{1}$ and $T_{2}$, are alternatively connected to $V_{1}, V_{2}$, and $V_{3}$. A sequence of measurements as discussed in the text yields correction for the phase shifter and the transducer. The inverter is assumed to be perfect.)

Fig. 8, self-contained calibration of the phase shifter and the power transducer is possible at $\pm 90^{\circ}$. If the power transducer is linear-that is, the phase angle corrections at leading and lagging power factors are identical-only two measurements are required. For the first the connection is as in Fig. 8; for the second, the connections to the power transducer are reversed. The sum of the two power readings and the difference between them are proportional to the phase-angle corrections of the phase shifter and the power transducer, respectively.

Electronic transducers may not have identical corrections at $\pm 90^{\circ}$ and $-90^{\circ}$. In such circumstances, a minimum of three, preferably four, measurements are required. The inverter must be ideal with respect to the phase angle. Let us define $\beta$ as the quadrature correction of the phase shifter, and $a$ and $b$ the quadrature corrections of the power transducer in leading and lagging configurations, respectively. By using the four connections between the phase shifter/inverter and the power transducer as indicated below, certain proportionalities exist between the power readings and the phase-angle corrections:

$$
\begin{array}{ll}
V_{1} \text { to } T_{1}, V_{2} \text { to } T_{2} & P_{1}=-\beta-a \\
V_{1} \text { to } T_{2}, V_{2} \text { to } T_{1} & P_{2}=-\beta+b \\
V_{1} \text { to } T_{1}, V_{3} \text { to } T_{2} & P_{3}=+\beta+b \\
V_{1} \text { to } T_{2}, V_{3} \text { to } T_{1} & P_{4}=+\beta-a .
\end{array}
$$

Equations (4) and (7) are solved for $\beta$ and $a$; and (5) and (6) yield a value for $b$ and a redundant value for $\beta$. Such a test was performed, but with less than fully satisfactory results. Two types of difficulties were encountered: 1) the available power transducer, while within its specifications and quite satisfactory for its intended use, had peak-to-peak noise of about $20 \mu \mathrm{rad}$; and 2) for the particular experimental setup used, the phase shifter appeared to have a quadrature bias of $10 \mathrm{ppm}$. The definitive verifications of the self-testing procedure has yet to be carried out, nevertheless the theory appears sound.

The following power readings were obtained: $P_{1}=-44$ $\times 10^{-6}, P_{2}=93 \times 10^{-6}, P_{3}=68 \times 10^{-6}$, and $P_{4}=$ $-51 \times 10^{-6}$. These yielded the quadrature corrections of $a=-47.5 \mathrm{ppm}, b=80 \mathrm{ppm}, \beta=+3.5 \mathrm{ppm}$, and another value of $\beta=-12 \mathrm{ppm}$. The corresponding quadrature corrections of the power transducer as obtained with the digital phase-angle standard were $a=-30 \mathrm{ppm}$, and $b=65 \mathrm{ppm}$.

\section{Applications}

The instrument was designed and built for the specific purpose of calibrating voltage dividers and power transducers as used in the test equipment for measuring transformer losses. In this application the primary concern is to achieve very low phase-angle uncertainty.

Experience within the past year has shown that the ratio stability can be maintained within about $100 \mathrm{ppm}$ if some temperature control $\left( \pm 1^{\circ} \mathrm{C}\right)$ is available in the laboratory. Thus the divider can be used as a standard to calibrate metering-type voltage transformers and other moderately accurate dividers. The phase shifter, if combined with a stable transconductance amplifier [8], could serve as a quadrature volt-ampere source, for example to calibrate meters having standard inputs of $120 \mathrm{~V}$ and $5 \mathrm{~A}$. The phase shifter could also be used to suppress the quadrature signal to a low-power-factor power transducer, thus increasing the power factor as detected by the transducer. Direct measurement of loads having exceptionally low power factors, such as shunt reactor, power capacitors, and cables, might be feasible with moderately accurate power transducers.

\section{Conclusions}

The relatively simple instrument as described has proven to be a very versatile tool in calibrating and aligning, on a routine basis, test equipment that is used to measure power losses at low power factors. Much attention has been paid to have a self-contained system that would require minimal calibrations from outside. The results presented in this paper demonstrate that predictions of system performance given earlier [1] are valid.

While the circuit is deceptively simple, work at the accuracy levels involving microradians is never trivial. Effects that are negligible at 1-percent level could completely invalidate the results at the 10-ppm level. Full appreciation of the operation of the system and awareness of sources of error is just as essential as any system of comparable accuracy.

\section{ACKNOWLEDGMENT}

The authors gratefully acknowledge the assistance of numerous colleagues, both at the National Bureau of Standards and ASEA Electric, in the completion of this project: A. G. Perrey constructed the electronics; and J. J. Nemec performed most of the tests. Additional technical and manuscript preparation assistance was received from N. M. Oldham, W. E. Anderson, J. C. Palla, M. E. Parker, and R. H. Palm. 


\section{REFERENCES}

[1] O. Petersons and S. P. Mehta, "Calibration of test systems for measuring power losses of transformers," Nat. Bur. Stand. Tech. Note 1204, Gaithersburg, MD, 1985.

[2] S. P. Mehta and O. Petersons, "Calibration of test systems for measuring power losses of transformers," presented at IEEE/PES Winter Meeting, Paper No. 86 WM 147-3, Feb. 1986.

[3] P. Miljanic, B. Stojanovic and P. Bosnjakovic, "The development of a high precision time-division power meter," in Dig. IEEE Conf. Precision Electromagnetic Measurements (Delft, the Netherlands), pp. 6768,1984

[4] E. So, "The application of the current comparator in instrumentation for high voltage power measurements at very low power factors,"' presented at "IEEE/PES Summer Meeting," Paper no. 85 SM 317-3, July 1985.

[5] N. L. Kusters and W. J. M. Moore, "The development and performance of current comparators for audio frequencies," IEEE Trans. Instrum. Meas., vol. IM-14, no. 4, pp. 178-190, Dec. 1965.

[6] O. Petersons, "A self-balancing current comparator," IEEE Trans. Instrum. Meas., vol. IM-15, no. 1, 2, Mar.-June 1966.

[7] N. M. Oldham, O. B. Laug, and B. C. Waltrip, "Digitally synthesized power calibration source," in Dig. 1986 Conf. Precision Electromagnetic Measurements (Gaithersburg, MD), 1986, p. 20.

[8] O. B. Laug, "A wide-band transconductance amplifier for current calibration," IEEE Trans. Instrum. Meas., vol. IM-34, no. 4, pp. 639643, Dec. 1985. 